\title{
The dynamic response of forest vegetation to hydro- thermal conditions in the Funiu Mountains of western Henan Province
}

\author{
ZHU Wenbo, LI Shuangcheng \\ College of Urban and Environmental Sciences, Peking University, Laboratory for Earth Surface Processes of \\ the Ministry of Education, Beijing 100871, China
}

\begin{abstract}
This paper uses $\mathrm{HJ}-1$ satellite multi-spectral and multi-temporal data to extract forest vegetation information in the Funiu Mountain region. The S-G filtering algorithm was employed to reconstruct the MODIS EVI (Enhanced Vegetation Index) time-series data for the period of 2000-2013, and these data were correlated with air temperature and precipitation data to explore the responses of forest vegetation to hydrothermal conditions. The results showed that: (1) the Funiu Mountain region has relatively high and increasing forest coverage with an average EVI of 0.48 over the study period, and the EVI first shows a decreasing trend with increased elevation below $200 \mathrm{~m}$, then an increasing trend from 200-1700 m, and finally a decreasing trend above $1700 \mathrm{~m}$. However, obvious differences could be identified in the responses of different forest vegetation types to climate change. Broad-leaf deciduous forest, being the dominant forest type in the region, had the most significant EVI increase. (2) Temperature in the region showed an increasing trend over the 14 years of the study with an anomaly increasing rate of $0.27^{\circ} \mathrm{C} / 10 \mathrm{a}$; a fluctuating yet increasing trend could be identified for the precipitation anomaly percentage. (3) Among all vegetation types, the evergreen broad-leaf forest has the closest EVI-temperature correlation, whereas the mixed evergreen and deciduous forest has the weakest. Almost all forest types showed a weak negative EVI-precipitation correlation, except the mixed evergreen and deciduous forest with a weak positive correlation. (4) There is a slight delay in forest vegetation responses to air temperature and precipitation, with half a month only for limited areas of the mixed evergreen and deciduous forest.
\end{abstract}

Keywords: forest vegetation; hydro-thermal condition; response time lag; EVI; Funiu Mountains, China

\section{Introduction}

Mountain ecosystems usually show complex environmental gradients and are sensitive to

Received: 2016-10-22 Accepted: 2016-12-21

Foundation: National Natural Science Foundation of China, No.41671090; National Basic Research Program (973 Program), No.2015CB452702

Author: Zhu Wenbo (1989-), PhD Candidate, specialized in the mountain ecosystem service, development and utilization of regional natural resources. E-mail: zhuwb517@163.com

"Corresponding author: Li Shuangcheng (1961-), Professor, mainly engaged in the ecosystem services.

E-mail: scli@urban.pku.edu.cn 
climate change. As a result of global climate change, they have become the focus for studying the dynamic responses of vegetation to climate change (Xiao et al., 2005; Wang et al., 2011; Zhu et al., 2011; Yao et al., 2015). Following the vertical differentiation of mountain climate, evergreen broad-leaf forest, broad-leaf deciduous forest, coniferous forest, and various other vegetation types appear sequentially from mountain base to mountain top (Zhang et al., 2002). An investigation into the relationship between forest vegetation types and climate change enhances our understanding of the complexity and dynamics of mountain ecosystems and reveals the mechanisms of their response to climate change.

Previous studies have discovered a close relationship between mountain vegetation changes and precipitation, and a significant difference among the various vegetation types to hydrothermal conditions (Neigh et al., 2008; Yang et al., 2011; Zhang et al., 2011; Dai et al., 2015; Wang et al., 2016). Cui et al. analyzed the altitudinal variation of air temperature and vegetation coverage in the Qinling Mountain region, China, and found out that high altitude areas have the highest correlation with air temperature (Cui et al., 2013). Wang et al. revealed a one-month delay for vegetation growth in response to precipitation in China's southern hilly and mountainous regions (Wang et al., 2014). Miao et al. suggested that vegetation in the Mongolian Plateau demonstrates a balanced and heterogeneous response to climate change, and that grassland vegetation is positively correlated with precipitation (Miao et al., 2014). Nevertheless, mountain vegetation classification used in these studies only includes forest and grassland; detailed classification of forest vegetation is still lacking. Moreover, research on the relationship between the coverage of various vegetation types and climate is only concentrated in plateau regions (Zhang et al., 2011; Bao et al., 2013; Wang et al., 2014; Zhang et al., 2015). In addition, these studies often take Normalized Difference Vegetation Index (NDVI) to assess vegetation coverage changes. However, NDVI is too sensitive to vegetation canopy background, prone to saturation, and considerably affected by soil noise. On the other hand, Enhanced Vegetation Index (EVI), derived from improved NDVI, allows higher monitoring accuracy and better reflects vegetation growth conditions (Huete et al., 2002). The use of EVI to investigate the relationships between forest vegetation type and air temperature and precipitation is absolutely necessary for further exploration of the balance and heterogeneity of mountain environments.

The Funiu Mountain region, located in the transitional zone between subtropical and warm temperate zones in central China and characterized by complex flora compositions, is an ideal site for mountain research (Ma et al., 2004; Zhang et al., 2016). But, research work has been mainly focused on surveying the flora diversity and the biome characteristics of the region (Ding et al., 2006; Fan et al., 2008), with some research exploring the relationship between dynamic variations in the mountain forest landscape and topographic gradient ( $\mathrm{Li}$ ang et al., 2010) or with the relationship between vegetation dynamics and climatic factors. This research utilized $30 \mathrm{~m}$ multi-temporal data from the HJ-1 satellite to extract forest vegetation types in the Funiu Mountain region, and applied the S-G filtering algorithm to reconstruct the time-series MODIS EVI data from 2000 to 2013. Integrating these data with the climatic observation data, the spatial and temporal variation characteristics of the regional forest types and their relationships with air temperature and precipitation were analyzed. 


\section{Data source and method}

\subsection{Background of the research area}

The Funiu Mountains extend from northwest to southeast in the western Henan Province of China. Located between $110^{\circ} 30^{\prime} \mathrm{E}-113^{\circ} 30^{\prime} \mathrm{E}, 32^{\circ} 45^{\prime} \mathrm{N}-34^{\circ} 20^{\prime} \mathrm{N}$, they belong to the eastern part of the Qinling Mountain Range (Figure 1), spanning 8 counties: Luanchuan, Songxian, Lushan, Xixia, Neixiang, Zhenping, Lushi, and Nanzhao. The mean annual temperature is $13.6^{\circ} \mathrm{C}-15.1^{\circ} \mathrm{C}$, with an annual precipitation of 700-1000 mm. The Funiu Mountain region is located in the transitional zone between subtropical and warm temperate areas, also with outstanding altitudinal differences in physical geographical characteristics. Accordingly, its vegetation belongs to the transitional type between warm temperate broad-leaf deciduous forest and northern subtropical mixed evergreen and deciduous forest.

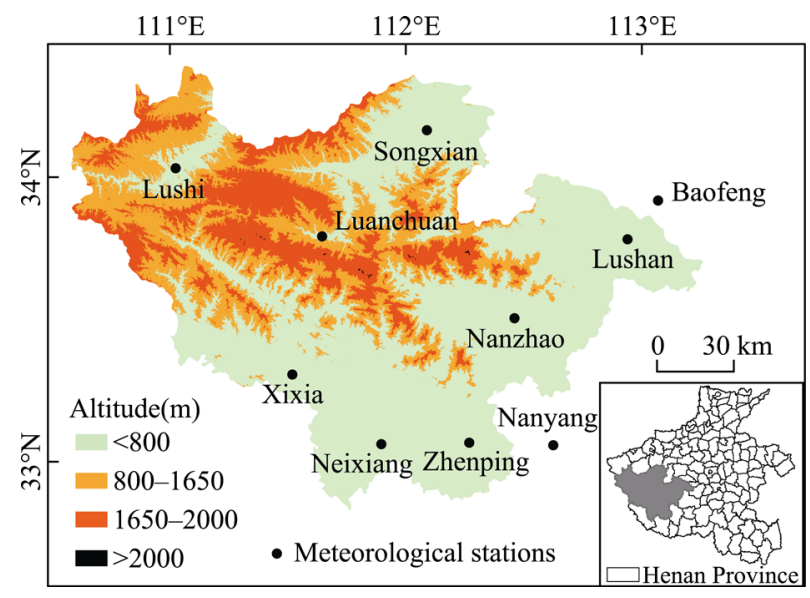

Figure 1 The location and topography of the Funiu Mountains, western Henan Province

\subsection{Data source}

\subsubsection{Remote sensing data}

The MOD13Q1 data were from the MODIS 2000-2013 data set, obtained from the National Aeronautics and Space Administration Office (NASA), with a spatial resolution of $250 \mathrm{~m}$ and a temporal resolution of 16 days. First, EVI data were extracted from the MOD13Q1 raw data set with the MRT tool and subjected to re-projection. In order to obtain better time series curves, the Savitzky-Golay filter was employed to re-project the MODIS EVI 16-day images for fitting the EVI variation (Chen et al., 2009). For the forest vegetation classification, cloudless and clear HJ-1 satellite $30 \mathrm{~m}$ multi-spectral data for January, April, and October of 2015 were employed. With ENVI 5.0, the data were subjected to various treatments, such as atmospheric correction, geometric correction, mosaic, and cropping into the research area. Altitude data were derived from the $30 \mathrm{~m}$ DEM of the ASTER GDEM V2, from the International Scientific Data Mirror Site of the Computer Network Information Center, Chinese Academy of Sciences.

\subsubsection{Climatic data}

Plant growth is influenced by the hydrothermal conditions and responds with a certain time delay to air temperature and precipitation (Cui et al., 2009; Shen et al., 2015). Air temperature and precipitation were selected in order to study the EVI response of various forest vegetation types to climatic factors. The air temperature and precipitation data were taken from 19 climatic stations during 1991-2013, of which 14 stations were from the Chinese Climatic Scientific Data Sharing Site and 5 from the Henan Climatic Bureau (Figure 1). The growing 
growing season of forest vegetation in the Funiu Mountain region is from May to September. Based on the daily mean air temperature and precipitation during the growing seasons of 1991-2013, the annual mean temperature and precipitation for the growing season of each year was obtained in order to study the variation characteristics of air temperature anomalies and precipitation anomaly percentages with time. According to the biweekly mean temperature and the biweekly precipitation from 2000 to 2013, the annual variation patterns of these two parameters were also analyzed. These parameters were compared with EVI changes within a year to see if they show any consistency. Based on the monthly mean temperature and precipitation during growing seasons from 2000 to 2013, the relationship between annual EVI and climatic factors was investigated. Furthermore, the delay in the response of biweekly EVI during the growing season to climatic factors was investigated based on the biweekly mean temperature and precipitation during March to September in 2000-2013.

\subsection{Research method}

\subsubsection{Forest vegetation classification}

In remote sensing images, objects of the same type may show different spectra under observation whereas some objects of different classes may have the same spectral curves. To reduce the classification errors brought about by these phenomena, multi-temporal remote sensing images were employed. In order to avoid "salt and pepper effects" due to pixel classification, the object-oriented classification method of the eCognition software was used to extract forest types. Forest vegetation in the Funiu Mountains was therefore classified into 5 types: evergreen broad-leaf forest, evergreen coniferous forest, deciduous coniferous forest, deciduous broad-leaf forest, and mixed evergreen and deciduous forest (Figure 2). To verify the classification accuracy, 120 sampling points were randomly chosen to assess the accuracy of the vegetation survey data in the study area. The results suggested an overall accuracy of $84.2 \%$ for the forest classification and a kappa coefficient of 0.8 . The on-site survey discovered that forests in the mountain areas require a relatively long succession period. Therefore, in the past 14 years, the spatial variation ranges for the different vegetation classes in the region have been limited, and the vegetation class data can therefore be employed for effective research.

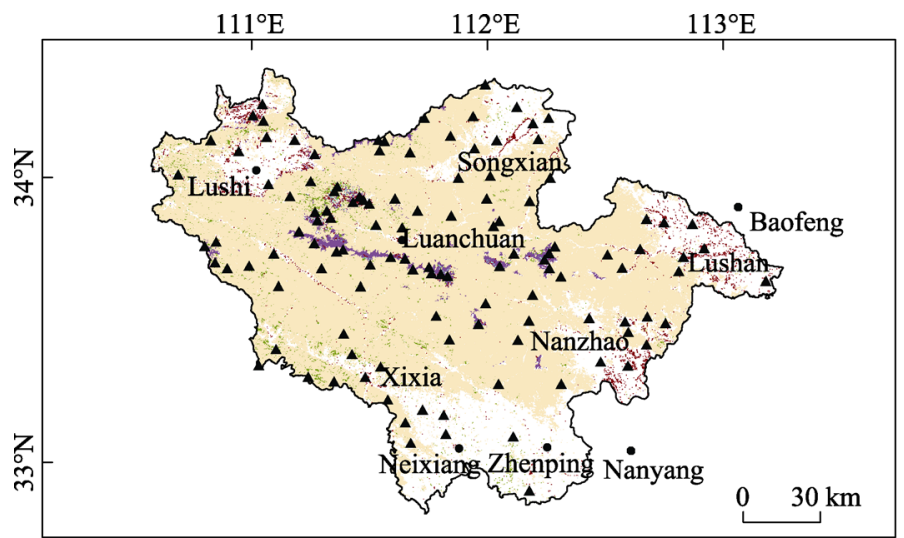

- Sampling points for accuracy assessment

Evergreen-and-deciduous mixed forest

Evergreen coniferous forest Deciduous broad-leaf forest Deciduous coniferous forest Evergreen broad-leaf forest Study area

Figure 2 Vegetation types in the Funiu Mountains 


\subsubsection{Definition of forest vegetation growing seasons}

The complete vegetation growth cycles were obtained by integrating the EVI data over the past 14 years. TIMESAT software was used to extract the pixel-based phenology information and confirm the average growing season of forest vegetation during 2000-2013. The vegetation index obtained from remote sensing images showed noise interference. Hence, TIMESAT was applied for noise reduction and vegetation index fitting, in order to obtain a time series of vegetation index and to extract vegetation phenology information (Jönsson et al., 2002, 2004) (Figure 3). It can be shown that $50.76 \%$ of the study area showed the growing season starting at 105th-120th days, i.e., in late April (Figure 3a), and that, $46.85 \%$ of the study area showed the growing season closing at 285th-300th days or in late October. (Figure 3b). Therefore, by integrating the spatial and temporal distribution of large-scale leaf growth and withering, the growing season for the vegetation in the Funiu Mountain region was defined as May to September.
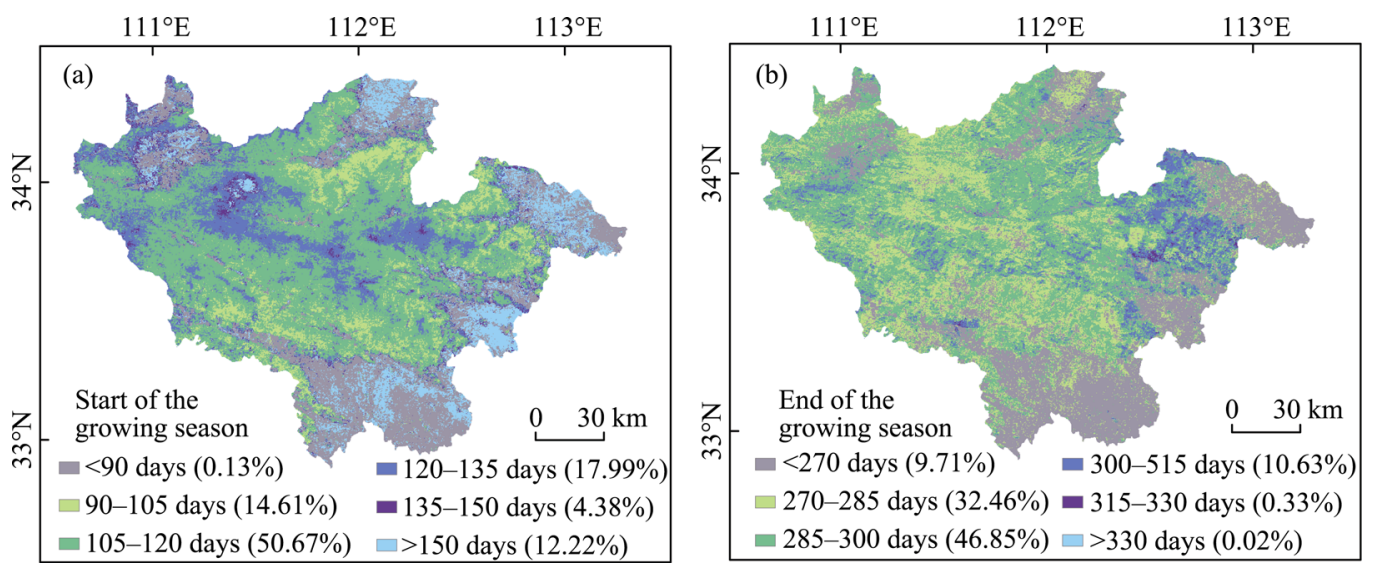

Figure 3 Spatial distribution of (a) the start and (b) the end of the vegetation growing season in the Funiu Mountain region

\subsubsection{Spatial interpolation method for climatic data}

With the thin-plate smoothing spline function, the associated factors were taken as covariates. Spatial interpolation of climatic data was performed with ANUSPLIN, which better enhances the spatial accuracy of time series climatic data interpolation (Liu et al., 2008). Compared with ordinary kriging, inverse-distance weighting, and the thin-plate smoothing spline function method, the thin-plate smoothing spline interpolation method (ANUSPLIN) gives the best interpolation results for air temperature and precipitation, in particular for complex mountain environments (Yu et al., 2008). Hence, ANUSPLIN was selected for air temperature and precipitation data interpolation to obtain the biweekly mean temperature and precipitation raster data, with a pixel resolution of $250 \mathrm{~m}$.

\subsubsection{Linear trends and correlation analysis}

For the 2000-2013 EVI trend analysis, the slope of the linear regression equation was used to indicate the spatial and temporal characteristics of the vegetation. This method reflects the vegetation patterns through the trend analysis on the raster data consisting of pixels ( $\mathrm{Xu}$ et al., 2002). 


$$
\theta=\frac{n \times \sum_{i=1}^{n} i \times E V I_{i}-\left(\sum_{i=1}^{n} i\right)\left(\sum_{i=1}^{n} E V I_{i}\right)}{n \times \sum_{i=1}^{n} i^{2}-\left(\sum_{i=1}^{n} i\right)^{2}}
$$

where $\theta$ is the slope of the trend line; $n$ is the research period; $i$ is the order of research period; $E V I_{i}$ is the EVI of the ith growing season. if $\theta>0$, it represents an increasing trend; if $\theta<0$, it represents a decreasing trend. An F-test was carried out to verify the significance of the variation trend. With the $\theta$ values and the F-test results, the yearly variation trends of the growing season EVI were divided into 5 classes (Mu et al., 2013): decreasing very significantly $(\theta<0, P<0.01)$; decreasing significantly $(\theta<0,0.01<P<0.05)$; no obvious changes $(P>0.05)$; increasing significantly $(\theta>0,0.01<P<0.05)$; and increasing very significantly $(\theta>0, P<0.01)$.

Using ENVI/IDL, the pixel-based correlation analysis was conducted and the Pearson correlation coefficient was calculated. Significance verification was also carried out. In order to better reflect the correlation between vegetation and climatic factors in the research area, correlation coefficients were classified based on the degree of correlation: highly correlated $(1 r \mathrm{l} \geqslant 0.8)$; moderately correlated $(0.5 \leqslant 1 r l<0.8)$; poorly correlated $(0.3 \leqslant 1 r l<0.5)$; and weakly correlated $(0 \leqslant 1 r 1<0.3)$.

\section{Results}

\subsection{Spatial and temporal characteristics of EVI}

The growing season EVI from 2000 to 2013 in the Funiu Mountain region is approximately $0.39-0.58$, with an overall average value of 0.48 . It is high in the central area but low near the boundaries (Figure 4a). By comparing Figure 4a with Figure 2, it can be seen that the forest vegetation EVI is generally higher, $0.35-0.68$, in the central areas, whereas the shrubs, meadows, and crops near the mountain bases at the research area boundaries have a lower EVI. Analysis of the altitude-vegetation EVI relationship shows that the EVI first shows a decreasing trend with elevation below $200 \mathrm{~m}$, then an increasing trend from 200-1700 m, and followed by a decreasing trend with elevation above $1700 \mathrm{~m}$ (Figure 4b). The highest vegetation EVI is observed at 1600-1700 m whilst the lowest is below $200 \mathrm{~m}$. This is related to the vertical distribution of the vegetation (Song et al., 1994). Below $800 \mathrm{~m}$ are mainly low-mountain shrubs, meadows, and crops with relatively low EVI values. At 800-1650 m, deciduous broad-leaf forest, with a relatively high EVI, is often present. At 1650-2000 m, mixed coniferous broad-leaf forest dominates. Above $2000 \mathrm{~m}$, there are mostly high-mountain shrubs and krummholz. Hence, the EVI gradually decreases after $1650 \mathrm{~m}$.

The significance analysis on the annual variation in growing season EVI for 2000-2013 in the Funiu Mountain region (Figure 5) demonstrates that $33.19 \%$ of the research area shows a very significantly increasing trend, $21.29 \%$ shows a significantly increasing trend, $44.72 \%$ has no obvious changes, $0.38 \%$ shows a significantly decreasing trend, and $0.42 \%$ shows a very significantly decreasing trend respectively. The areas with an increasing EVI trend $(54.48 \%)$ far exceed those with a decreasing trend $(0.4 \%)$. This suggests that the 
growing season EVI shows an overall stable increase over the past 14 years at the $90 \%$ confidence interval. The dominant deciduous broad-leaf forest shows the most considerable increase. From the spatial distribution (Figure 5), the areas with a significantly and very significantly decreasing EVI and those with a significantly increasing EVI are scattered over the research area. The areas with no obvious changes are mostly located in the regions with non-forest vegetation, whereas the areas with a very significant EVI increase are mainly those of deciduous broad-leaf forest.
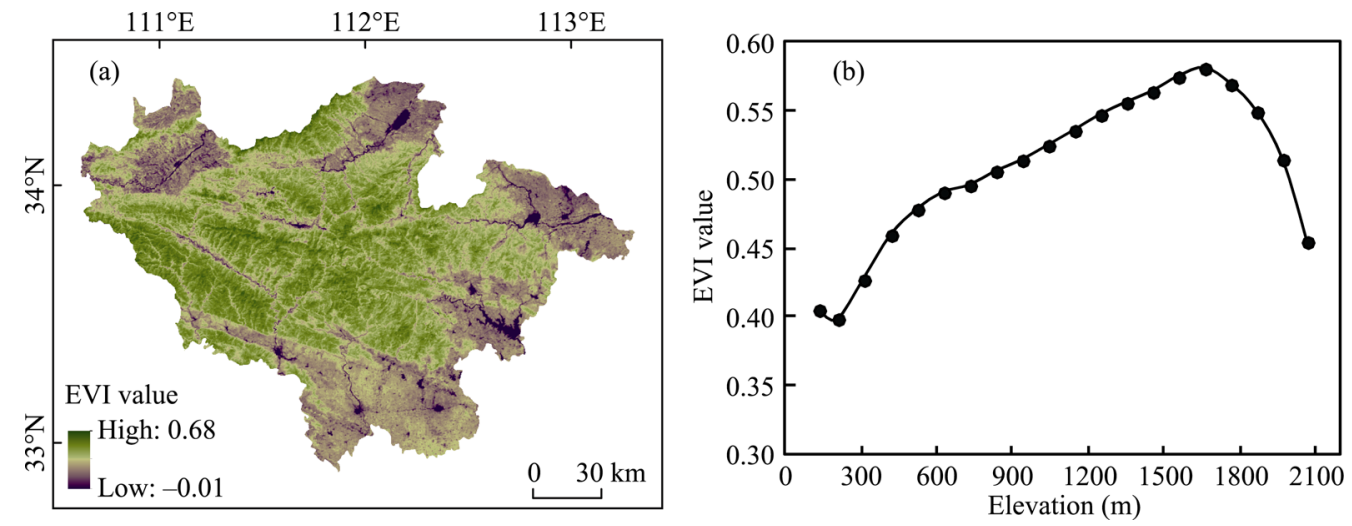

Figure 4 Spatial distribution (a) and vertical characteristics (b) of mean growing season EVI in the Funiu Mountains from 2000 to 2013
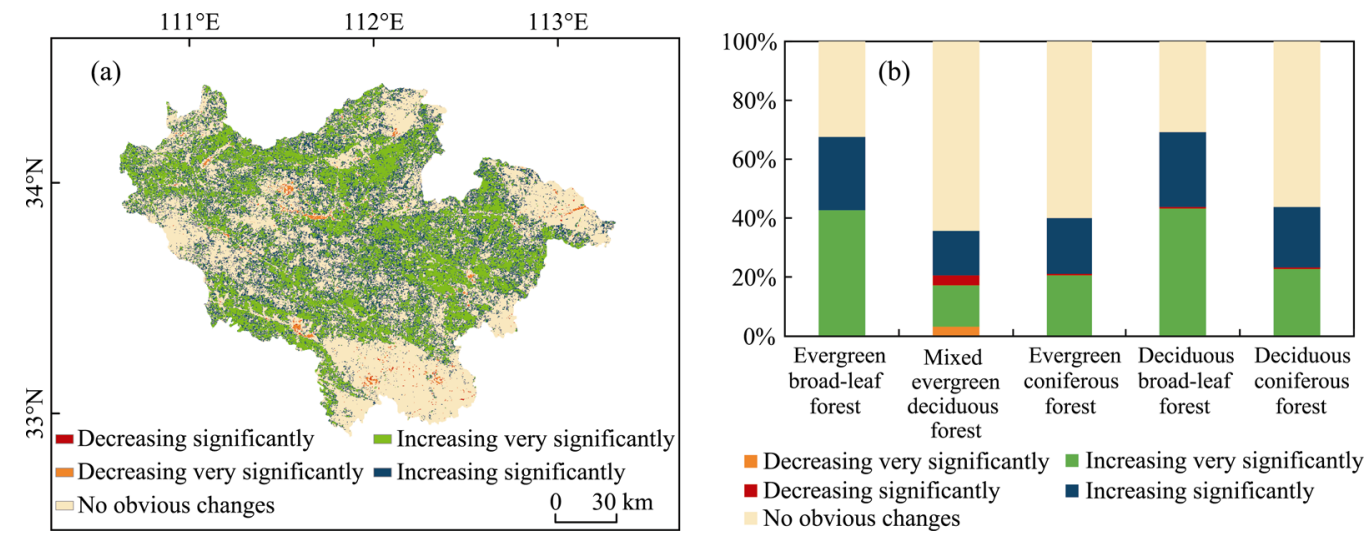

Figure 5 Spatial distribution (left) and area proportion results (right) of significance test of EVI annual changes for various forest vegetation types in the Funiu Mountains from 2000 to 2013

\subsection{Variation in climatic factors}

As a basic aridity index, the precipitation anomaly percentage can reflect the arid condition caused by precipitation variability (Yan et al., 2012). Data from 8 weather stations in the study area were selected to analyze the growing season precipitation anomaly percentage and the air temperature anomaly variations during 1991-2013 in the region (Figure 6a). The results indicate that the precipitation anomaly proportion is relatively small in the 1990s and gradually increases after 2000. In general, growing season precipitation in the Funiu Mountain region shows a gradually increasing trend over the last 21 years. From the 1991-2013 
growing season air temperature anomaly graph, the anomaly is relatively low in 1991-1993 and then the anomaly increases slowly (positive trend line slope) at a rate of $0.27^{\circ} \mathrm{C} / 10 \mathrm{a}$, revealing a warming trend in this region (Figure $6 b$ ).
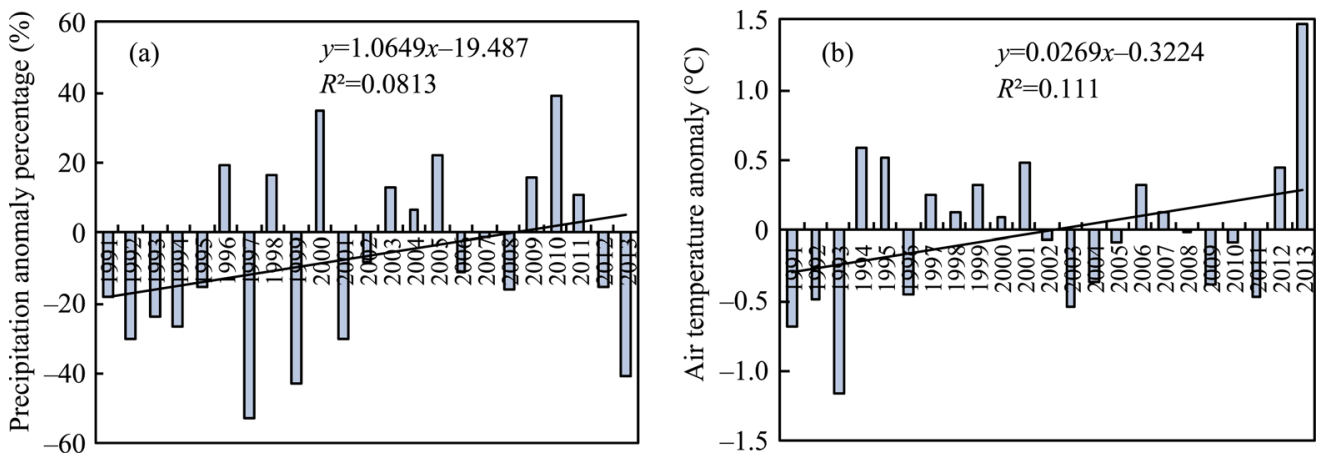

Figure 6 Trends of precipitation anomaly percentage (a) and temperature anomaly (b) in the Funiu Mountains from 1991-2013

\subsection{Variation of biweekly vegetation EVI and climatic factors}

The variations in average biweekly EVI with time (Figure 7) show that EVI from January to July increases gradually and then decreases after reaching its peak in July. Vegetation EVI is greater than 0.3 during May to September. In particular, EVI variations are greater in March and October. This is because large-scale growth starts in the dominant deciduous broad-leaf forest during late March to early April. In May, the new leaves of the deciduous trees become relatively lush and the forest vegetation coverage is higher, increasing the overall EVI. In July, the EVI starts to diminish gradually after reaching its peak. At the late stage of the growing season in September to October, the leaves of the dominant deciduous forest start to fall. The EVI then decreases and falls below 0.3 after October. Both the biweekly mean air temperature and biweekly precipitation in a year increase at first and then decrease. Both reach their peaks in July and then drop gradually. This indicates that the climatic hydrologic parameters are in phase with the thermal parameters in the study area.

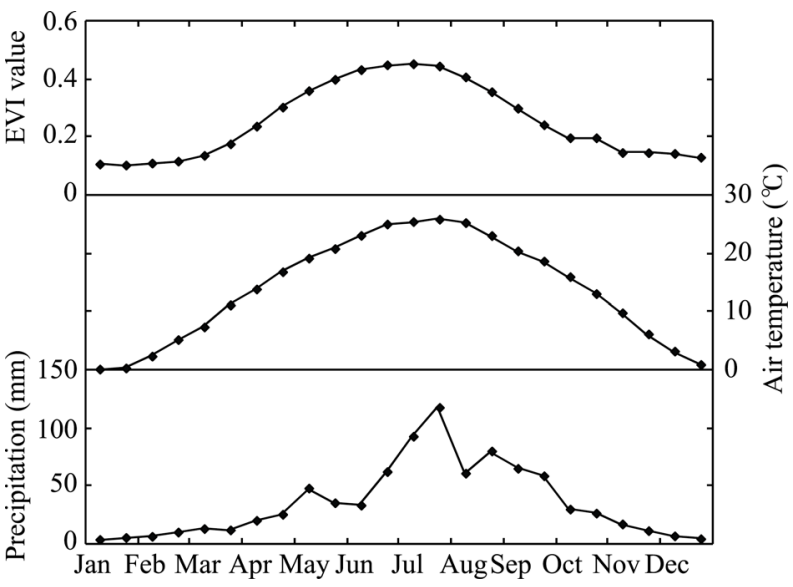

Figure 7 Biweekly changes in vegetation EVI, mean temperature, and precipitation in the Funiu Mountains

\subsection{Relationship between growing season EVI variations and climatic factor varia- tions}

The correlation between the annual growing season EVI and air temperature and precipitation was analyzed in the Funiu Mountain region and subjected to significance verification 
(Figure 8). The area of regions with significant EVI-air temperature correlation is 3099.75 $\mathrm{hm}^{2}, 14.28 \%$ of the total study area. The area of regions with significant EVI-precipitation correlation is $792.13 \mathrm{hm}^{2}, 3.65 \%$ of the total area. The regions with significant EVI-air temperature correlation are mainly located in the southwest whereas those with significant EVI-precipitation correlation are fewer and mostly located near the boundaries. There are therefore considerable differences between the EVI-air temperature correlation spatial distribution and that of the EVI-precipitation correlation. The EVI-air temperature correlation is stronger in the west than in the east (Figure 8a), mostly because the western forest has higher coverage and is more sensitive to air temperature variations. The EVI-precipitation correlation is stronger at low altitudes than at high altitudes (Figure 8b). Due to considerable human activity and dominant farmland and grassland, the low-altitude areas are more easily affected by hydrological factors.
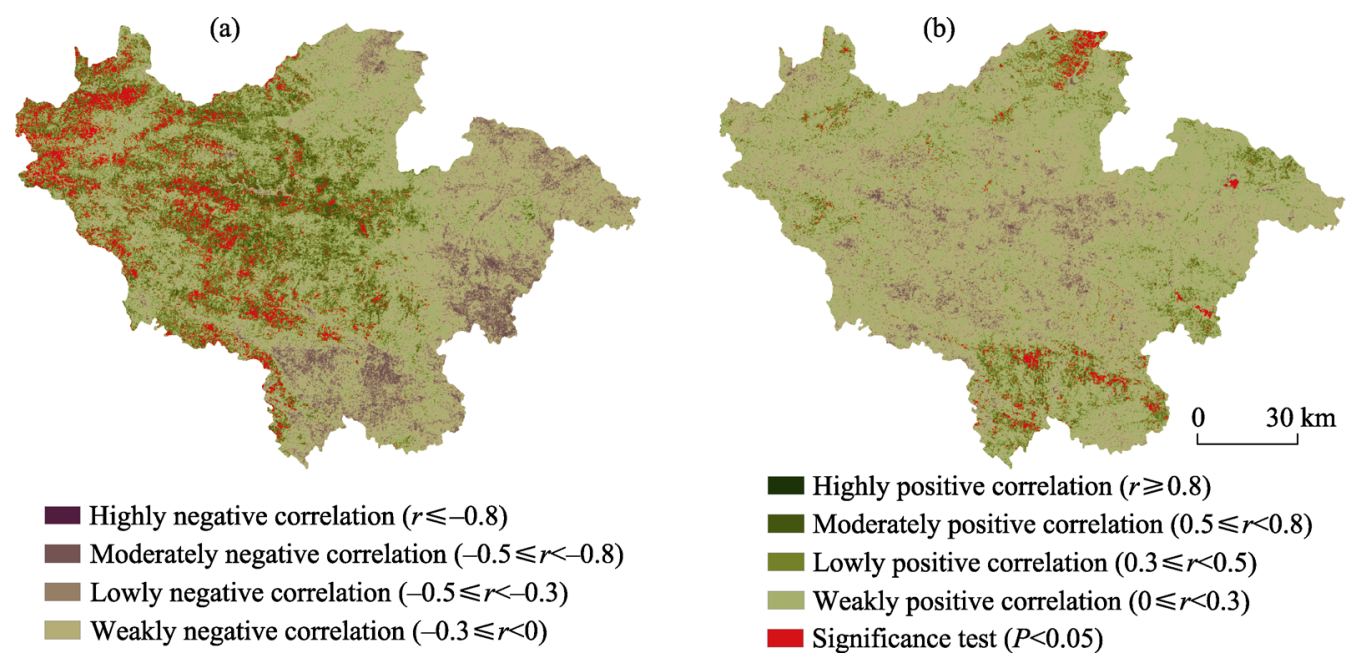

Figure 8 The spatial distribution of significance test results and correlation coefficient for (a) mean EVI and temperature and (b) mean EVI and precipitation in the Funiu Mountains during the growing seasons of 2000-2013

The EVI-air temperature and precipitation correlation coefficients of different forest vegetation types were investigated and their coverage areas and percentages were calculated, respectively (Table 1). There are considerable differences between the correlation relationships of various vegetation types. The evergreen broad-leaf forest has the highest EVI-air temperature correlation. Some $19.03 \%$ and $37.48 \%$ of the total area show a moderate positive correlation $(0.5 \leqslant r<0.8)$ and a low positive correlation $(0.3 \leqslant r<0.5)$, respectively. With the second highest correlation, $34.66 \%$ of the evergreen coniferous forest area and $34.08 \%$ of the deciduous coniferous forest area show low positive correlations. Some $27.09 \%$ and $35.78 \%$ of the deciduous broad-leaf forest area show low positive and weak positive EVI-temperature correlations, respectively. The EVI-temperature correlation for mixed evergreen deciduous forest is not obvious. Some $19.97 \%$ of this forest suggests a weak positive correlation $(0 \leqslant r<0.3)$ while $31.31 \%$ indicates a weak negative correlation $(-0.3 \leqslant r<0)$. The EVI-precipitation correlation also differs for different forest vegetation types. $43.26 \%$ of the mixed evergreen coniferous forest has an EVI positively but weakly correlated with precipi- 
tation $(0 \leqslant r<0.3)$, while $44.81 \%$ of the evergreen coniferous forest, $52.12 \%$ of the evergreen broad-leaf forest, $50.93 \%$ of the deciduous coniferous forest and $50.27 \%$ of the deciduous broad-leaf forest show a weak negative correlation between EVI and precipitation $(-0.3 \leqslant r<0)$.

Table 1 The area and proportion of correlation between EVI and temperature, precipitation within forest vegetation types in the Funiu Mountains during growing season

\begin{tabular}{|c|c|c|c|c|c|c|}
\hline $\begin{array}{c}\text { Correlation } \\
\text { coefficient class }\end{array}$ & & $\begin{array}{l}\text { Mixed } \\
\text { evergreen } \\
\text { deciduous } \\
\text { forest }\end{array}$ & $\begin{array}{c}\text { Evergreen } \\
\text { coniferous } \\
\text { forest }\end{array}$ & $\begin{array}{l}\text { Evergreen } \\
\text { broad-leaf } \\
\text { forest }\end{array}$ & $\begin{array}{c}\text { Deciduous } \\
\text { coniferous } \\
\text { forest }\end{array}$ & $\begin{array}{l}\text { Coniferous } \\
\text { broad-leaf } \\
\text { forest }\end{array}$ \\
\hline \multirow{2}{*}{ Highly positive } & Air temperature & 0.05 & 0.23 & 0.25 & 0.35 & 0.11 \\
\hline & Precipitation & - & - & - & - & 0 \\
\hline \multirow{2}{*}{ Moderately positive } & Air temperature & 6.57 & 17.44 & 19.03 & 17.87 & 14.46 \\
\hline & Precipitation & 1.29 & 0.18 & 0.28 & 0.26 & 0.28 \\
\hline \multirow{2}{*}{$\begin{array}{l}\text { Low positive } \\
\text { correlation }\end{array}$} & Air temperature & 14.88 & 34.56 & 37.48 & 34.08 & 27.09 \\
\hline & Precipitation & 11.91 & 3.18 & 3.75 & 1.89 & 3.25 \\
\hline \multirow{2}{*}{ Weak positive } & Air temperature & 19.97 & 39.96 & 34.75 & 36.95 & 35.78 \\
\hline & Precipitation & 43.26 & 22.7 & 33.69 & 19.54 & 35.44 \\
\hline \multirow{2}{*}{ Weak negative } & Air temperature & 31.31 & 7.29 & 7.25 & 9.55 & 2.42 \\
\hline & Precipitation & 34.16 & 44.81 & 52.12 & 50.93 & 50.27 \\
\hline \multirow{2}{*}{ Low negative } & Air temperature & 20.57 & 0.46 & 1.06 & 1.06 & 17.59 \\
\hline & Precipitation & 7.31 & 24.18 & 9.17 & 22.83 & 9.78 \\
\hline \multirow{2}{*}{ Moderately negative } & Air temperature & 6.61 & 0.05 & 0.18 & 0.15 & 2.32 \\
\hline & Precipitation & 2.07 & 4.94 & 0.92 & 4.9 & 0.98 \\
\hline \multirow{2}{*}{ Highly negative } & Air temperature & - & - & - & - & 0.23 \\
\hline & Precipitation & - & - & - & 0.02 & 0 \\
\hline
\end{tabular}

\subsection{Response delay of growing season biweekly EVI and climatic factor variations}

The correlated relationships between the growing season (May to September) biweekly EVI and the air temperature and precipitation for: the same period; two weeks earlier; 1 month earlier; 1.5 months earlier; and 2 months earlier were analyzed and subjected to significance verification (Figure 9). The monthly EVI-temperature and precipitation correlation and the correlation significance show the same variations. The correlations, in descending order, are: in the same period $>$ two weeks earlier $>1$ month earlier $>1.5$ months earlier $>2$ months earlier. In the F test, the correlation coefficients with both EVI and temperature and precipitation taken in the same period have the greatest areas with acceptable significance. On the other hand, the correlation with temperature and precipitation measured 2 months before the growing season has the smallest area with acceptable significance.

With the ENVI/IDL program, the air temperature and precipitation correlation coefficient images were subjected to multi-band synthesis. The locations with the maximum coefficients were extracted and marked (Figure 10). The regions with an EVI response delay are mostly near the boundaries. Lag time is often two weeks or a month and there is no response 
lag time of 1.5 or 2 months. In order to investigate the characteristic lag time of EVI in response to air temperature and precipitation for different forest vegetation types, the lag time spatial distributions for air temperature and precipitation, respectively, were overlapped with the forest vegetation map, and a statistical survey was carried out (Table 2). The 5 vegetation types show identical patterns. Most of the study area has no response delay, whereas that with a 2-week lag time has the second largest percentage. The area with a 1-month lag time is the smallest. Some $73.14 \%$ and $85.09 \%$ of the mixed evergreen deciduous forest have immediate responses to air temperature and precipitation, respectively. More than $99 \%$ of evergreen coniferous forest, evergreen broad-leaf forest, deciduous coniferous forest, and

(a) In the same period
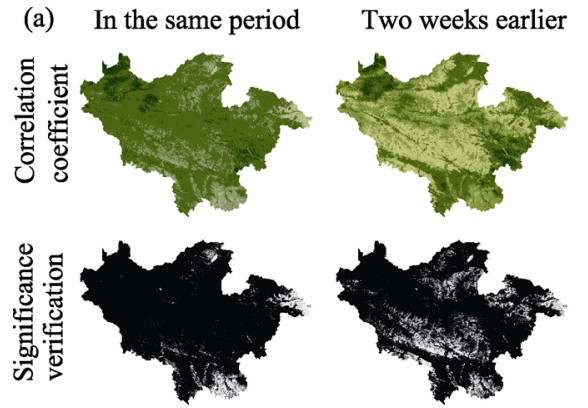

(b)
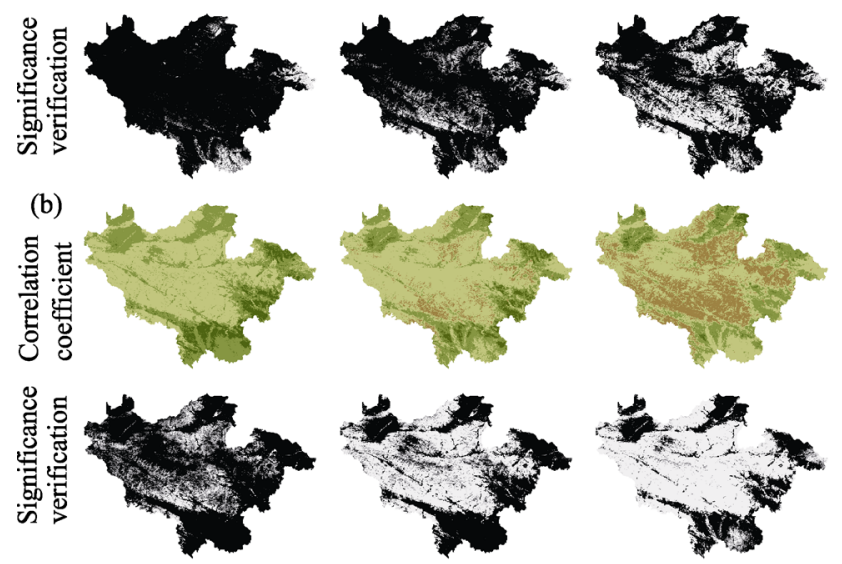
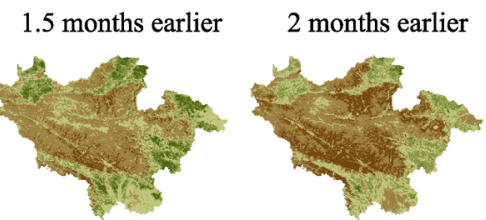

Highly positive correlation $(r \geqslant 0.8) \quad$ Moderately positive correlation $(0.5 \leqslant r<0.8)$
Weak positive correlation $(0 \leqslant r<0.3) \quad$ Weak negative correlation $(-0.3 \leqslant r<0)$

Weak positi $P<0.005$

$0.005<P<0.01$
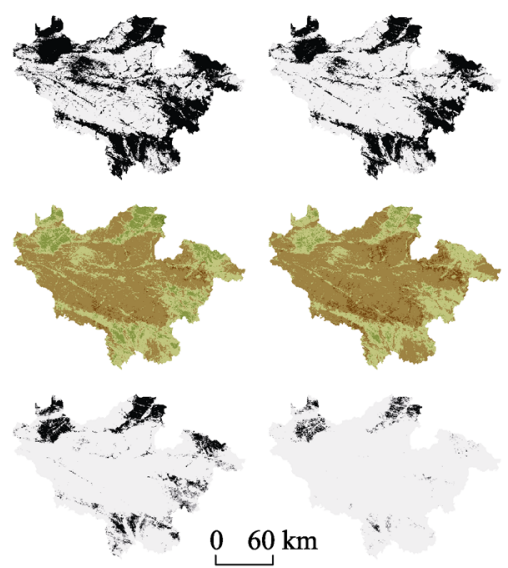

Figure 9 The spatial distribution of correlation coefficient and significance test results between EVI and temperature (a), and EVI and precipitation (b) in the Funiu Mountains during the growing season
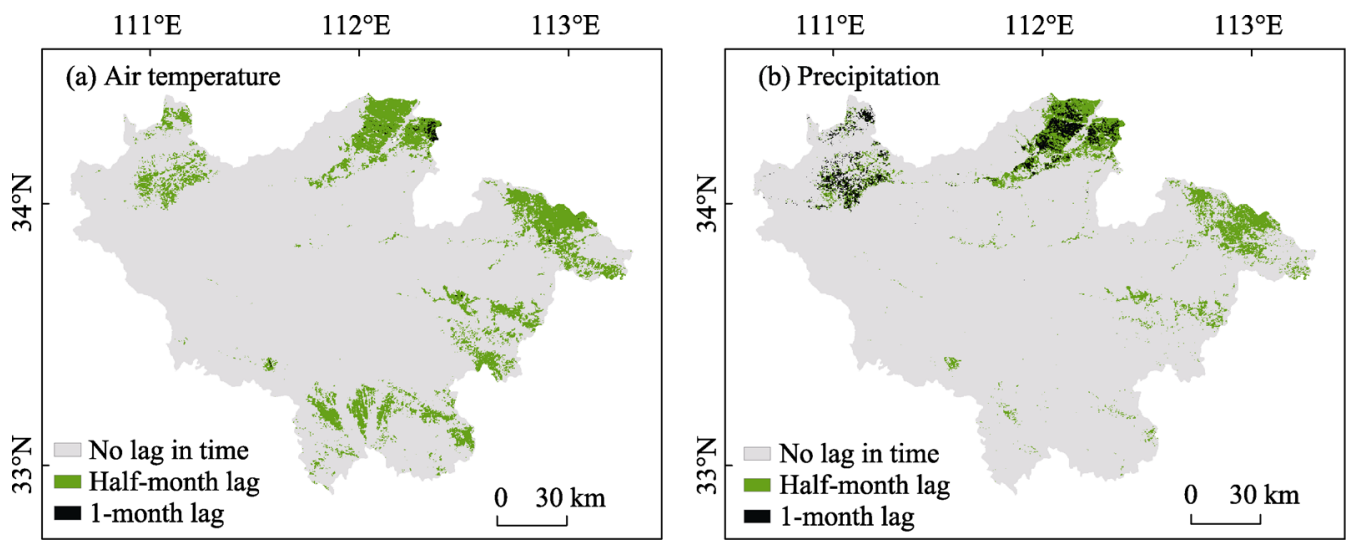

Figure 10 The spatial distribution of response lag of EVI to temperature (a) and precipitation (b) in the Funiu Mountains 
deciduous broad-leaf forest show no delay in responding to air temperature and precipitation. The areas with 2-week and 1-month lag time constitute relatively small proportions of the 5 forest vegetation types. Compared with the other 4 vegetation types, mixed evergreen deciduous forest has a greater area with a 2 -week response delay. The results suggest that, in general, the forest vegetation types in the Funiu Mountain region do not show a delay in their response to air temperature and precipitation, and evergreen deciduous forest shows a 2-week lag only in limited areas.

Table 2 The area percentage of different response delays to temperature and precipitation for various forest types in the Funiu Mountains

\begin{tabular}{llccccc}
\hline Lag Time & & $\begin{array}{c}\text { Mixed ever- } \\
\text { green de- } \\
\text { ciduous forest }\end{array}$ & $\begin{array}{c}\text { Evergreen } \\
\text { coniferous } \\
\text { forest }\end{array}$ & $\begin{array}{c}\text { Evergreen } \\
\text { broad-leaf } \\
\text { forest }\end{array}$ & $\begin{array}{c}\text { Deciduous } \\
\text { coniferous } \\
\text { forest }\end{array}$ & $\begin{array}{c}\text { Coniferous } \\
\text { broad-leaf } \\
\text { forest }\end{array}$ \\
\hline \multirow{2}{*}{ No lag } & Air temperature & 73.14 & 99.77 & 99.24 & 99.7 & 99.65 \\
& Precipitation & 85.09 & 99.35 & 99.17 & 99.67 & 99.33 \\
\hline \multirow{2}{*}{ Half-month lag } & Air temperature & 27 & 0.23 & 0.8 & 0.3 & 0.41 \\
& Precipitation & 12.97 & 0.74 & 0.78 & 0.11 & 0.65 \\
\hline \multirow{2}{*}{ 1-month lag } & Air temperature & 0.04 & - & 0.04 & - & 0.01 \\
& Precipitation & 2.14 & 0.28 & 0.12 & 0.11 & 0.08 \\
\hline
\end{tabular}

\section{Conclusions}

This paper investigated the dynamic, regional-scale forest responses to climate change in the Funiu Mountains and provides fundamental scientific data and technical support for monitoring and forecasting changes in mountain forest systems. However, due to remote sensing image resolution, interpolation accuracy, and inadequate survey information, there are certain difficulties in analyzing the structural responses of vegetation to air temperature and precipitation. In future, high-resolution images and fixed-point observations should be integrated so as to analyze the responses of various vegetation types to air temperature and precipitation at both community and species scales. Furthermore, different methodologies and data should be employed to establish models for vegetation dynamics and hydrothermal changes. This will allow study of vegetation responses to climate change at larger spatial and temporal scales, which is fundamental to future research.

Based on time series of MODIS EVI data reconstructed by the S-G filtering algorithm and air temperature and precipitation data, correlation analyses were conducted to study the spatial and temporal characteristics of the response of various forest vegetation types to air temperature and precipitation during the period of 2000-2013 in the Funiu Mountain region. The conclusions are as follows:

(1) The EVI first increases with altitude but then decreases at high altitude. Its peak is located at an elevation of 1600-1700 m. Areas with increasing EVI (54.48\%) are far larger than those with decreasing values $(0.4 \%)$. Most of the areas with increasing EVI belong to deciduous broad-leaf forest. This shows that forests are in a state of succession toward healthy development in the Funiu Mountain region.

(2) The increasing rate of air temperature is approximately $+0.27^{\circ} \mathrm{C} / 10 \mathrm{a}$ in the study area for recent years. The precipitation anomaly percentage shows a fluctuating yet increasing 
trend. The correlation between forest vegetation EVI and air temperature is closer than that between EVI and precipitation. The EVI-temperature correlation for evergreen broad-leaf forest is the highest. Evergreen coniferous forest and deciduous coniferous forest have the second closest EVI-temperature correlation. Mixed evergreen and deciduous forest has the weakest. Only the mixed evergreen and deciduous forest has a weak positive EVI-precipitation correlation; all other vegetation types show mainly weak negative correlations.

(3) The monthly EVI has the highest correlation with monthly air temperature and precipitation in most areas where the correlation coefficients pass the significance verification $(P<0.05)$. In general, there is no obvious delay in forest response to air temperature and precipitation in the Funiu Mountains, only a 2-week delay in some areas of the mixed evergreen and deciduous forests.

\section{References}

Bao Gang, Bao Yuhai, Qin Zhihao et al., 2013. Vegetation cover changes in Mongolian Plateau and its response to seasonal climate changes in recent 10 years. Scientia Geographica Sinica, 33(5): 613-621. (in Chinese)

Chen J, Jönsson P, Tamura M et al., 2004. A simple method for reconstructing a high-quality NDVI time-series data set based on the Savitzky-Golay filter. Remote Sensing of Environment, 91(3): 332-344.

Cui Linli, Shi Jun, Yang Yinming et al., 2009. Ten-day response of vegetation NDVI to the variations of temperature and precipitation in eastern China. Acta Geographica Sinica, 64 (7): 850-860. (in Chinese)

Cui Xiaolin, Bai Hongying, Wang Tao, 2013. Difference in NDVI with altitudinal gradient and temperature in Qinling area. Resources Science, 35(3): 618-626. (in Chinese)

Dai L, Feng Y X, Luo G P et al., 2015. The relationship between soil, climate and forest development in the mid-mountain zone of the Sangong River watershed in the northern Tianshan Mountains, China. Journal of Arid Land, 7(1): 63-72.

Ding Shengyan, Lu Xunling, 2006. Comparison of plant flora of Funiu Mountains and Jigong Mountain natural reserves. Geographical Research, 25(1): 62-70. (in Chinese)

Fan Yulong, Hu Nan, Ding Shengyan et al., 2008. A study on the classification of plant functional types based on the dominant herbaceous species in forest ecosystem at Funiu Mountains national natural reserve. Acta Ecologica Sinica, 28(7): 3092-3101. (in Chinese)

Huete A, Didan K, Miura T et al., 2002. Overview of the radiometric and biophysical performance of the MODIS vegetation indices. Remote Sensing of Environment, 83(1): 195-213.

Jönsson P, Eklundh L, 2002. Seasonality extraction by function fitting to time-series of satellite sensor data. IEEE Transactions on Geoscience and Remote Sensing, 40(8): 1824-1832.

Jönsson P, Eklundh L, 2004. TIMESAT: A program for analyzing time-series of satellite sensor data. Computers \& Geosciences, 30(8): 833-845.

Liang Guofu, Han Yan, Ding Shengyan, 2010. Forest landscape dynamics in north of Funiu Mountains along terrain gradient. Scientia Geographica Sinica, 30(2): 242-247. (in Chinese)

Liu Zhihong, Mc Vicar T R, Li Lingtao et al., 2008. Interpolation for time series of climatic variables using ANUSPLIN. Journal of Northwest A\&F University (Natural Science Edition), 36(10): 227-234. (in Chinese)

Ma Jianhua, 2004. Laws of soil vertical variations on southern slope of Funiu Mt.: Simultaneous study on north boundary of subtropical zone. Acta Geographica Sinica, 59(6): 998-1011. (in Chinese)

Miao Lijuan, Jiang Chong, He Bin et al., 2014. Response of vegetation coverage to climate change in Mongolian Plateau during recent 10 years. Acta Ecologica Sinica, 34(5): 1295-1301. (in Chinese)

$\mathrm{Mu}$ Shaojie, Li Jianlong, Zhou Wei et al., 2013. Spatial-temporal distribution of net primary productivity and its relationship with climate factors in Inner Mongolia from 2001 to 2010. Acta Ecologica Sinica, 33(12): 3752-3764. (in Chinese)

Neigh C S R, Tucker C J, Townshend J R G, 2008. North American vegetation dynamics observed with mul- 
multi-resolution satellite data. Remote Sensing of Environment, 112(4): 1749-1772.

Shen Xiangjin, Zhou Daowei, Li Fei et al., 2015. Vegetation change and its response to climate change in grassland region of China. Scientia Geographica Sinica, 35(5): 622-629. (in Chinese)

Song Chaoshu, 1994. Scientific Survey of the Funiu Mountains Nature Reserve. Beijing: China Forestry Publishing House, 10-11. (in Chinese)

Wang Can, Ren Zhiyuan, Zhang Chong, 2014. Intra-annual response of NDVI to changes in temperature and precipitation and its spatial characteristics in northern Shaanxi. Research of Soil and Water Conservation, 21(1): 173-177. (in Chinese)

Wang Genxu, Deng Wei, Yang Yan et al., 2011. The advances, priority and developing trend of alpine ecology. Journal of Mountain Science, 29(2): 129-140. (in Chinese)

Wang Jing, Wang Kelin, Zhang Mingyang et al., 2014. Temporal-spatial variation in NDVI and drivers in hilly terrain of Southern China. Resources Science, 36(8): 1712-1723. (in Chinese)

Wang Yuhang, Zhao Mingfei, Kang Muyi et al., 2016. Spatial scale-dependent and non-stationarity relationships between NDVI and climatic factors in the Loess Plateau. Geographical Research, 35(3): 493-503. (in Chinese)

Xiao J, Moody A, 2005. Geographical distribution of global greening trends and their climatic correlates: 1982-1998. International Journal of Remote Sensing, 26(11): 2371-2390.

Xu Jianhua. Mathematical Methods in Modern Geography, 2002. Beijing: Higher Education Press, 48-51. (in Chinese)

Yan Jianwu, Chen Baozhang, Fang Shifeng et al., 2012. The response of vegetation index to drought: Taking the extreme drought disaster between 2009 and 2010 in Southwest China as an example. Journal of Remote Sensing, 16(4): 720-737. (in Chinese)

Yang Z, Gao J, Zhou C et al., 2011. Spatio-temporal changes of NDVI and its relation with climatic variables in the source regions of the Yangtze and Yellow Rivers. Journal of Geographical Sciences, 21(6): 979-993.

Yao Yonghui, Zhang Baiping, 2015. The spatial pattern of monthly air temperature of the Tibetan Plateau and its implications for the geo-ecology pattern of the Plateau. Geographical Research, 34(11): 2084-2094. (in Chinese)

Yu Fei, Zheng Xiaobo, Gu Xiaoping et al., 2008. Comparative study on spatial interpolation of climate elements precision in complex mountainous environment. Journal of Guizhou Meteorology, 32(3): 3-6. (in Chinese)

Zhang Baiping, Yao Yonghui, Mo Shenguo et al., 2002. Digital spectra of altitudinal belts and their hierarchical system. Journal of Mountain Science, 20(6): 660-665.

Zhang Chong, Ren Zhiyuan, Yuan Xin, 2011. Intra-annual response of NDVI to changes in temperature and precipitation and its spatial characteristics in northwest China. Resources Science, 33(12): 2356-2361. (in Chinese)

Zhang Geli, Xu Xingliang, Zhou Caiping et al., 2011. Responses of vegetation changes to climatic variations in Hulun Buir grassland in past 30 years. Acta Geographica Sinica, 66(1): 47-58. (in Chinese)

Zhang Jinghua, Feng Zhiming, Jiang Luguang et al., 2015. Analysis of the correlation between NDVI and climate factors in the Lancang River Basin. Journal of Natural Resources, 30(9): 1425-1435. (in Chinese)

Zhang Jingjing, Wang Yansong, Zhu Lianqi et al., 2016. Study on change of northern subtropical border in mountainous regions in western Henan Province. Journal of Henan University (Natural Science), 46(1): 40-49. (in Chinese)

Zhu Lianqi, Xu Limin, 2011. Analysis of effects of global change on terrestrial ecosystem. Areal Research and Development, 30(2): 161-164. (in Chinese) 